\title{
Metastatic Carcinoma of Endometrium from Primary Ovarian Serous Carcinoma Mimicking a Primary Uterine Tumour
}

\author{
Mengfei $\mathrm{Xu}^{1}$, Feng $\mathrm{Zhou}^{1}$ and Lili Huang${ }^{2}$
}

\begin{abstract}
A 44-year female was admitted to the hospital, complaining of vaginal bleeding. Ultrasound imaging revealed two masses in the pelvic cavity, measuring $6.6 \times 3.4 \times 1.8 \mathrm{~cm}$ and $8.2 \times 4.7 \times 3.7 \mathrm{~cm}$ in size. After surgical debulking of the tumours, the patient underwent abdominal hysterectomy, bilateral salpingo-oophorectomy, pelvic disease dissection, appendicectomy, omentectomy, and pelvic lymph node dissection. Histological and immunochemical examinations confirmed the diagnosis of serous carcinomas in both ovaries involving the right tubal fimbria, the omentum, pelvic nodules, and the endometrium. Ovarian serous carcinoma spreading to the endometrium is a rare phenomenon and may mimick a primary uterine serous cercinoma. Although difficult, it is important to distinguish concomitant independent primary tumours from metastases because the most appropriate management strategy and prognosis differs in both.
\end{abstract}

Key Words: Endometrial carcinoma, Ovarian carcinoma, Metastatic disease.

\section{INTRODUCTION}

Concomitant development of endometrial and ovarian cancer is somewhat infrequent but not rare; and synchronous primary carcinoma of the endometrium and ovary (SCEO), primary endometrial carcinoma with ovarian metastasis, and primary ovarian carcinoma with endometrial metastasis are, thereof, the three principal forms. Approximately, $5 \%$ of patients diagnosed with uterine cancer have a concomitant ovarian carcinoma, and the reciprocal incidence is approximately $10 \% .{ }^{1}$ It is diagnostically challenging to definitively distinguish a primary cancer combined with a metastasis from two independent primary cancers; especially, when the histological subtypes are identical. Relevant pathological criteria were first proposed by Ulbright and Roth. ${ }^{2}$

Here, we report a rare case of bilateral primary serous carcinoma of ovary with endometrial metastasis to facilitate resolution of diagnostic problems.

\section{CASE REPORT}

A 44-year (gravidity 2, parity 2) female was admitted complaining of vaginal bleeding for 2 months. She had neither significant past medical history, nor any family history of carcinoma. Physical examination revealed

Department of Pathologyl / Obstetrics and Gynecology², Women's Hospital, School of Medicine, Zhejiang University, 1 Xueshi Road, Hangzhou, Zhejiang, China

Correspondence: Dr. Feng Zhou, Department of Pathology, Women's Hospital, School of Medicine, Zhejiang University, 1 Xueshi Road, Hangzhou, Zhejiang, China

E-mail: fungchew@zju.edu.cn

Received: August 15, 2018; Accepted: October 23, 2018 bilateral adnexae with solid masses. The uterus was of normal size, not tender on palpation, and poorly active. The serum levels of cancer antigen (CA) 125 and CA $15-3$ were 372.5 and $41.4 \mathrm{U} / \mathrm{mL}$, respectively. Ultrasound revealed heterogeneous masses in both posterior uterine parametria, indistinguishable in terms of imaging charac-teristics from those of the ovaries; the masses were $6.6 \times 3.4 \times 1.8 \mathrm{~cm}$ and $8.2 \times 4.7 \times 3.7 \mathrm{~cm}$ in size. After tumour debulking, the patient underwent abdominal hysterectomy, bilateral salpingo-oophorectomy, dissection of the pelvic disease site, appendicectomy, omentectomy, and pelvic lymph node dissection.

Macroscopically, uterine cavity showed a polypoid nodule measuring $1 \mathrm{~cm}$ in size, and a friable soft mass measuring $4 \times 3 \times 1 \mathrm{~cm}$ in size was evident on the uterine serosa. Multi-nodular friable pelvic masses measuring $6 \times 2.5 \times$ $2.5 \mathrm{~cm}$ and $7 \times 4 \times 4 \mathrm{~cm}$ in size were also evident, probably arising from both ovaries. The right tubal fimbria contained a lump measuring $1.5 \times 1 \times 1 \mathrm{~cm}$ in size. Large nodules were also evident on the intestinal surfaces; and on the omentum, all pelvic biopsy specimens yielded pathological data consistent with the presence of such nodules. The left fallopian tube was normal.

Microscopically, the endometrial mass was characterised by large areas of solid growth and focal areas exhibiting complex, confluent glandular proliferation. Stromal invasion was not prominent. Poorly defined islands (sheets or nests) of medium-sized tumour cells were apparent. These cells exhibited very high nucleao cytoplasmic ratios, rounded nuclei, clumped chromatin, prominent nucleoli, and high mitotic activity. The tumours of both ovaries, the right tubal fimbria, the uterine serosa and intestinal surfaces, and the omentum, and the pelvic nodules, were similar to the endometrial tumours. 

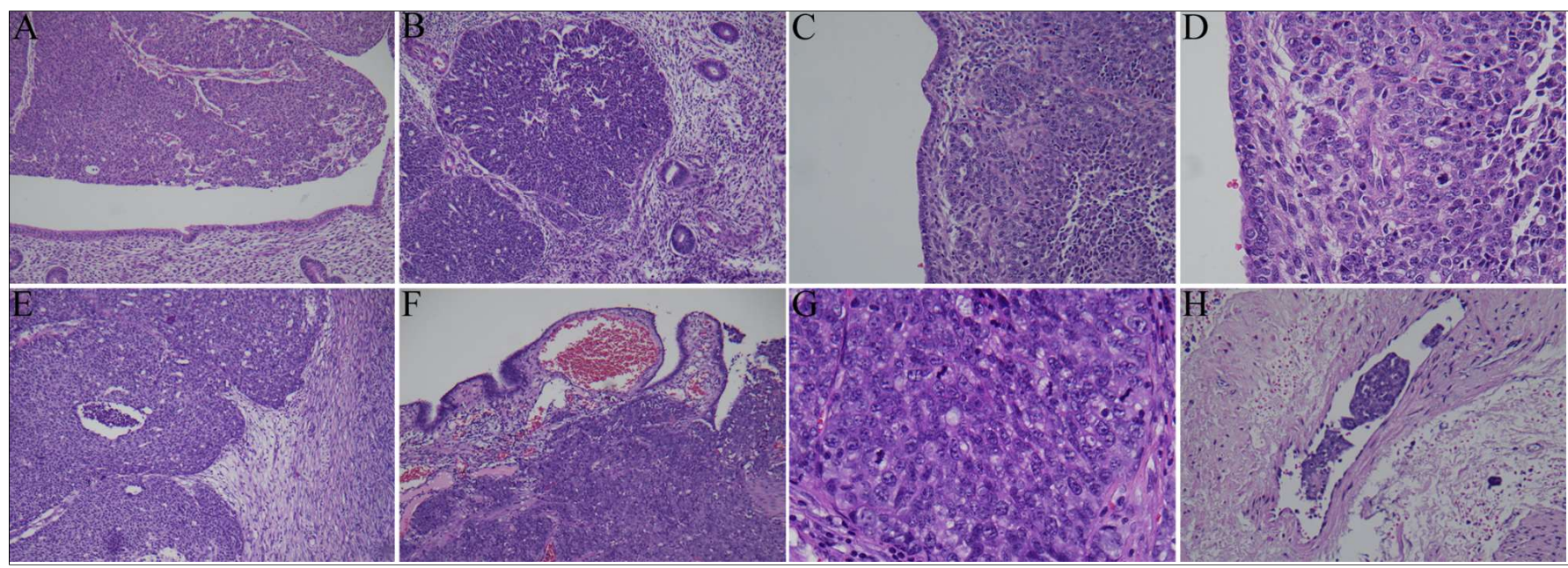

Figure 1: The endometrial tumour exhibited solid growth (A 100x) and fewer areas exhibiting complex, confluent glandular proliferation (B 100x). Stromal invasion was not prominent (A, B 100x). These atypical cells had high nuclear:cytoplasmic ratios, prominent nucleoli $(C 200 \times)$ and high-level mitotic activity( D $400 \times)$. The tumours of the ovaries (E 100x) and the right tubal fimbria (F 100x). Mitotic figures (G 400x) and LVSI can be seen (H 400x).

Lymphovascular space invasion (LVSI) was evident in the ovary and uterine serosa. Peritoneal cytology identified malignant cells. Four of eleven pelvic lymph nodes were involved (Figure 1).

Immunohistochemically, the endometrial and ovarian tumours were identical, being positive for Wilms' tumour gene 1 (WT1), p16, insulin-like growth factor-II mRNA binding protein 3 (IMP3), p53, and oestrogen receptor (ER) with high Ki67 index values.

\section{DISCUSSION}

Synchronous endometrial and ovarian tumours account for half of all synchronous female genital tract neoplasms. ${ }^{3}$ Regarding simultaneous neoplasms involving the endometrium and ovary, independently originating tumours are more common than secondary tumours. ${ }^{4}$ It is critical to distinguish SCEO from metastatic disease to define the precise tumour stage and make appropriate treatment decisions.

Pathological criteria may play a significant role in differential diagnosis, but sometimes it causes diagnostic dilemma. If only one ovary is involved, and both tumoural sites are well-differentiated or moderately differentiated, a tumour of dual origin may be involved. 5 The endometrial mass was a small polypoid nodule in this case, and mimicked a primary endometrial carcinoma. However, both the endometrial and ovarian tumours were poorly differentiated, without atypical endometrial hyperplasia. The ovarian tumours were large and located in the parenchyma, reflecting direct extension from the ovary, predominantly into the outer wall of the uterus. These characteristics suggest that the ovarian cancer was primary in nature.

In this ambiguous condition, molecular genetic approaches and the use of immunohistochemical markers may facilitate accurate diagnosis. Measures of microsatellite instability, PTEN mutational status, loss of chromosomal heterozygosity, and KRAS and $\beta$-catenin expression levels have been suggested to be useful for diagnosis. ${ }^{6}$ However, the diagnostic utility of these parameters remains unclear.

The origin of concurrent endometrial and ovarian tumours is generally determined by evaluating the immunohistochemical expression of the ER, WT1, p16, IMP3, and p53.7-9 ER reactivity is demonstrated in almost all the ovarian serous carcinomas, whereas most of the uterine serous carcinomas are ER negative.8,9 Moreover, expression of WT1 proves that the tumour originates from the ovary rather than the endometrium. ${ }^{10}$ Notably, p16 and IMP3 overexpression is more frequent in serous carcinomas. ${ }^{7}$ p53, a tumour suppressor gene, is typically overexpressed in serous carcinomas and is regarded as a surrogate marker of such carcinomas. ${ }^{11}$ In this case, the tumour cells were strongly positive for ER, WT1, p16, IMP3 and p53, typical of a serous carcinoma of the ovary.

Uterine serous carcinoma often involves the ovaries but ovarian serous carcinoma rarely spreads to the endometrium. ${ }^{9}$ However, LVSI was evident in the ovary and uterine serosa. These characteristics suggest that the ovarian cancer was primary in nature and spread via a myometrial lymphatic route. Total abdominal hysterectomy and bilateral salpingo-oophorectomy with comprehensive staging are recommended following debulking, as required. A terminal ovarian metastatic carcinoma requires chemotherapy.

In conclusion, ovarian serous carcinoma spread to the endometrium is rarer and may mimick a primary one. The distinction between a metastatic carcinoma and two independent primary tumours is important in terms of precise diagnosis, tumour staging, appropriate treatment, and outcome. Histological diagnostic criteria remain controversial. We describe this case to facilitate resolution of diagnostic problems. 


\section{REFERENCES}

1. Jain V, Sekhon R, Pasricha S, Giri S, Modi KB, Shrestha E, et al. Clinicopathological characteristics and prognostic factors of synchronous endometrial and ovarian cancers - a singleinstitute review of 43 cases. Int J Gynecol Cancer 2017; 27: 938-46.

2. Ulbright TM, Roth LM. Metastatic and independent cancers of the endometrium and ovary: A clinicopathologic study of 34 cases. Hum Pathol 1985; 16:28-34.

3. Saatli B, Yildirim N, Ozay AC, Koyuncuoglu M, Demirkan B, Saygili U. Synchronous tumours of the female genital tract: A 20-year experience in a single center. Ginekol Pol 2014; 85: 441-5.

4. Irving JA, Catasus L, Gallardo A, Bussaglia E, Romero M, Matias-Guiu X, et al. Synchronous endometrioid carcinomas of the uterine corpus and ovary: alterations in the beta-catenin (CTNNB1) pathway are associated with independent primary tumours and favorable prognosis. Hum Pathol 2005; 36:605-19.

5. Soliman PT, Slomovitz BM, Broaddus RR, Sun CC, Oh JC, Eifel PJ, et al. Synchronous primary cancers of the endometrium and ovary: A single institution review of 84 cases. Gynecol Oncol 2004; 94:456-62.
6. Bese T, Sal V, Kahramanoglu I, Tokgozoglu N, Demirkiran F, Turan $\mathrm{H}$, et al. Synchronous primary cancers of the endometrium and ovary with the same histopathologic type versus endometrial cancer with ovarian metastasis: A single institution review of 72 cases. Int J Gynecol Cancer 2016; 26:394-406.

7. Zhang Y, Garcia-Buitrago MT, Koru-Sengul T, Schuman S, Ganjei-Azar P. An immunohistochemical panel to distinguish ovarian from uterine serous papillary carcinomas. Int $J$ Gynecol Pathol 2013; 32:476-81.

8. Lin J, Xi C, Liu C, Wang Y, Liu Y, Ren C, et al. Immunostaining study on the expression of NF-kB, ER, and PR in ovarian serous carcinoma. Int J Gynecol Pathol 2017; 36:58-63.

9. Nofech-Mozes S, Khalifa MA, Ismiil N, Saad RS, Hanna WM, Covens A, et al. Immunophenotyping of serous carcinoma of the female genital tract. Mod Pathol 2008; 21:1147-55.

10. Ussakli C, Usubutun A, Dincer N, Dolgun A, Bülbül D, Isikdogan $Z$, et al. Serous versus high-grade endometrioid endometrial carcinoma: Immunohistochemistry of RFP is not useful for differentiation. Pol J Pathol 2016; 67:221-7.

11. Hatano Y, Fukuda S, Makino H, Tomita H, Morishige KI, Hara A. High-grade serous carcinoma with discordant p53 signature: report of a case with newinsight regarding high-grade serous carcinogenesis. Diagn Pathol 2018; 13:24.

........... 